\title{
Medidas da radiação ionizante do meio ambiente local com um detector de raios gama
}

\section{Measurements of local environmental ionizing radiation with a gamma ray detector}

Rodrigo Rezende Fernandes de Carvalho*, Inácio Malmonge Martin, Rafael Augusto Gomes, Marcelo Pego Gomes

\section{RESUMO}

Raios gama de baixa energia, até $10 \mathrm{MeV}$, é um tipo de radiação eletromagnética de alta frequência produzida geralmente por decaimentos radioativos e descargas elétricas presentes no meio ambiente. Essa radiação, cuja intensidade varia a cada localidade do planeta, pode causar danos aos seres vivos. Foram efetuadas medidas da radiação gama de $200 \mathrm{keV}$ até $10,0 \mathrm{MeV}$ no campus do Instituto Tecnológico de Aeronáutica - ITA, em São José dos Campos, São Paulo, Brasil. Também foram realizadas medidas dessa radiação no intervalo de 10 min, no meio ambiente com fontes radioativas (césio Cs-137, polônio Po-210 e estrôncio Sr90) colocadas sobre o cristal cintilador. Com o estudo da geração dos espectros em função da energia sempre no mesmo intervalo de tempo, foi possível determinar, para este local, a presença da radiação gama natural na interface solo-atmosfera.

Palavras-chave: Radiação ionizante, Raios gama, Detector de radiação gama.

\begin{abstract}
Low energy gamma rays up to $10 \mathrm{MeV}$ is a type of high frequency electromagnetic radiation produced by decay of radioactive elements and by electric discharges present in the environment. The intensity of this radiation varies according to the location on the planet. Measurements of gamma radiation from $200 \mathrm{keV}$ to $10.0 \mathrm{MeV}$ were carried out in the Technological Institute of Aeronautics - ITA campus, in São José dos Campos, São Paulo, Brazil. In addition, measurements of this radiation were made within 10 min in the environment with radioactive sources (cesium Cs-137, polonium Po-210, and strontium Sr-90) placed on the scintillator. A generation of the spectra was studied in function of the energy, always in the same time interval. Thus, it was possible to determine the presence of the natural gamma radiation in the soil-atmosphere interface for this energy range.
\end{abstract}

Keywords: Ionizing radiation, Gamma rays, Gamma radiation detector. 


\section{INTRODUÇÃO}

O espectro da radiação gama geralmente é produzido por elementos químicos radioativos, como urânio $\left({ }^{238} \mathrm{U}\right.$ e $\left.{ }^{235} \mathrm{U}\right)$, tório $\left({ }^{232} \mathrm{Th}\right)$ e potássio $\left({ }^{40} \mathrm{~K}\right)$, no qual apresentam comprimentos de onda relativamente baixos, na ordem de picômetros. Devido ao seu comprimento, o poder de penetração desses elementos no meio ambiente é grande ${ }^{1}$. Com a sua alta taxa de energia, essa radiação apresenta um efeito ionizante, podendo causar danos irreparáveis no núcleo celular dos seres vivos. Países como os Estados Unidos da América têm estudos avançados sobre a interação da radiação com moléculas do corpo humano causando, em alguns casos, doenças como o câncer. A produção da radiação ionizante está sempre associada às partículas alfa ou beta. Após a emissão desse tipo de radiação, muitas vezes o núcleo atômico sofre um processo de reorganização, isto é, de passagem de um estado excitado para outro de menor energia, dando origem a emissões eletromagnéticas. Dessa forma, é comum ter-se uma emissão alfa seguida por uma gama, ou uma emissão beta seguida por uma gama $^{2}$. É possível medir radiações gama de diferentes intensidades de acordo com a região em que se encontra o equipamento sobre a superfície terrestre.

A quantidade de elementos radioativos presente no solo varia de acordo com a região do planeta Terra ${ }^{3}$. Existem diferentes técnicas para efetuar medidas dessa radiação nesse intervalo de energia tanto no solo quanto no ar ou na água ${ }^{4}$. O objetivo deste trabalho foi medir a radiação gama de $200 \mathrm{keV}$ até $10,0 \mathrm{MeV}$ no campus do Instituto Tecnológico de Aeronáutica - ITA, em São José dos Campos, São Paulo, Brasil.

\section{METODOLOGIA}

$\mathrm{O}$ aparato experimental não calibrado cujo objetivo é medir a radiação ionizante presente no local foi fornecido pela empresa Ludlum Measurements. Se trata de um sistema portátil constituído por um cristal cintilador de Iodeto de Sódio de 3 polegadas de altura por 3 polegadas de diâmetro dopado com tálio. Para sua proteção, o equipamento é envolvido por uma fina camada de alumínio em formato cilíndrico. Foram utilizados também uma fotomultiplicadora (PM), circuito de fonte de energia (regulada para fornecer 1.700 VDC), e um sistema de aquisição de dados disponibilizado pela empresa Aware Eletronic Inc., EUA ${ }^{4}$. A aquisição de dados foi realizada através de um laptop.

O primeiro passo deste trabalho foi medir a radiação ambiental presente no campus do ITA em São José dos Campos, São Paulo, Brasil, possibilitando identificar os elementos radioativos como bismuto $\left({ }^{214} \mathrm{Bi}\right)$, potássio $\left({ }^{40} \mathrm{~K}\right)$ e tálio $\left({ }^{208} \mathrm{Tl}\right)^{5}$. Através do sistema (software e hardware) de aquisição de dados obtiveram-se gráficos da intensidade de radiação versus canal, com uma amostragem ampla de até 1024 canais $^{6}$. Em seguida, utilizando as fontes radioativas Cs-137, Po-210 e Sr-90 existentes no laboratório do ensino fundamental do ITA - de intensidade de radiação muito baixa, em torno de $10 \mu \mathrm{Sv} / \mathrm{h}$, não necessitando de nenhum protocolo de proteção, foi feita a correlação entre canal e energia da radiação gama. Logo, todos os gráficos apresentados neste trabalho foram reportados em intensidade (contagens) versus energia da radiação incidente. Essa transformação foi executada utilizando recursos dos softwares Excel e Origin, na versão 2015.

\section{RESULTADOS E DISCUSSÃO}

A calibração do cintilador gama com fontes radioativas foi efetuada para determinar a intensidade dessa radiação ionizante medida em função da energia no meio ambiente. No gráfico da Fig. 1 observa-se, para uma medida da radiação ambiente do campus do ITA, a intensidade em contagens por canal $\times 0$ número do canal fornecido pelo detector durante $10 \mathrm{~min}$ de amostragem.

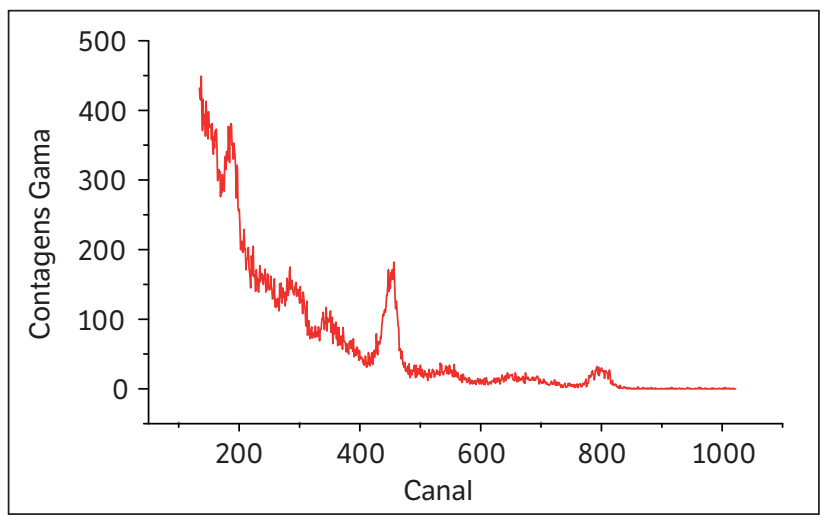

Figura 1: Gráfico da medição de raios gama presente no ambiente (intensidade $\times$ canal).

Para a melhor análise dos dados adquiridos foi efetuada, com auxílio das fontes radioativas e dos softwares Excel e Origin 2015, a transformação do gráfico de intensidade $\times$ canal para intensidade $\times$ energia (Fig. 2).

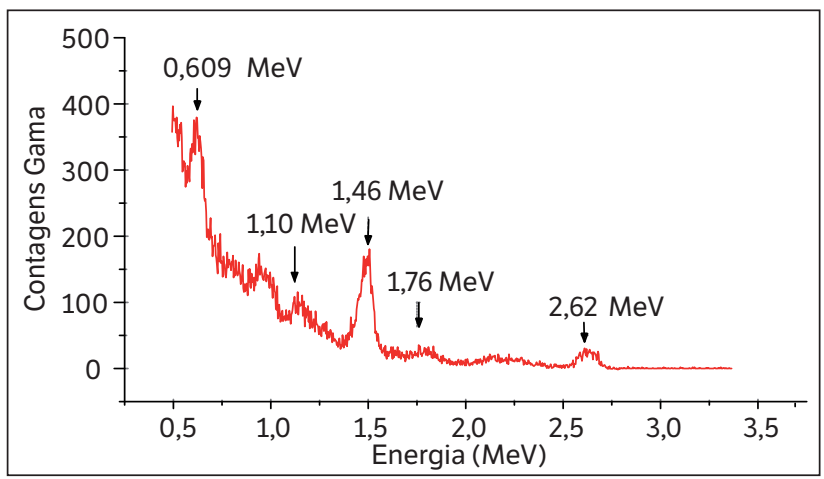

Figura 2: Gráfico da medição de raios gama presente no ambiente (intensidade $\mathrm{x}$ energia).

Na Fig. 2 o pico foto-absorção de 2,62 MeV indica a presença do elemento tório. Os picos de foto-absorção de 1,76 Mev, 1,10 Mev e 0,609 Mev indicam presença de urânio. O pico de foto-absorção de 1,46 MeV indica a presença de potássio. 
Em seguida foi efetuada uma nova medição e plotado o gráfico, utilizando como fontes radioativas o Cs-137 (emitindo raios gama), Sr-90 (emitindo particulas beta) e Po-210 (emitindo particulas alfa) posicionados em contato direto com o cintilador, como mostra a Fig. 3. Essas fontes fornecem energias de raios gama em 0,662 keV e 1,17 MeV, partículas alfas em 5,4 MeV e elétrons em $0,90 \mathrm{keV}$. $\mathrm{O}$ espectro resultante pode ser observado na Fig. 4.

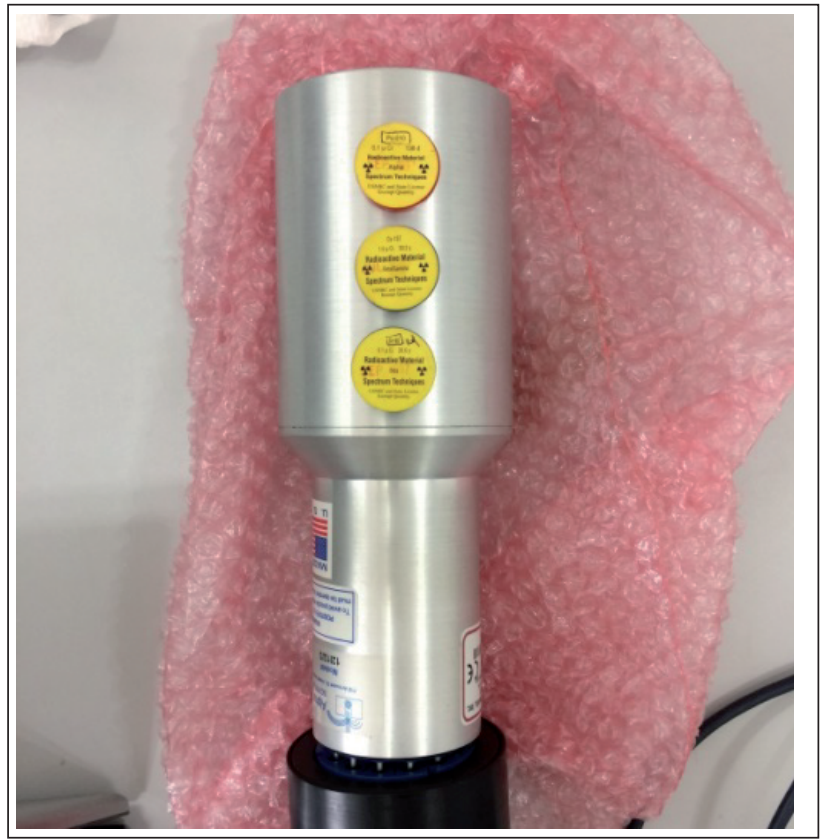

Figura 3: Foto do Gamma ray spectrometer em contato com os elementos radioativos Cs-137, Sr-90 e Po-210.

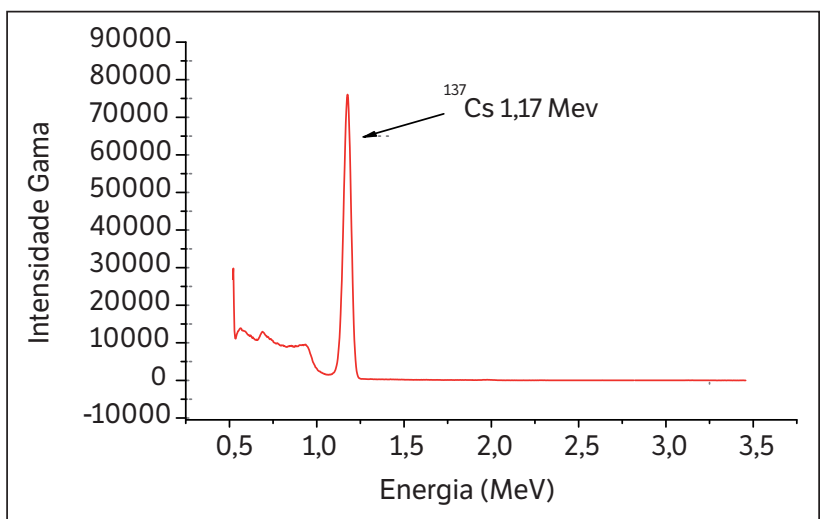

Figura 4: Grafico da medição da radiação emitida pelas fontes radioativas Cs-137, Sr-90 e Po-210 e pelo meio ambiente.

Em seguida, foi retirado o elemento radioativo Cs-137 e efetuada uma nova medição somente com os elementos emitindo partículas alfa e beta. O resultado obtido nesse procedimento é apresentado na Fig. 5.

Verifica-se na Fig. 5 que as partículas alfa e beta param no invólucro de alumínio do cristal e o espectro é o mesmo que o ambiental (Fig. 1). A última medição com o equipamento foi feita utilizando-se pequenas pedras que, se analisadas cuidadosamente, contêm Urânio em sua composição. Essas pedras, chamadas de uranitas, são supostamente originadas de vulcões e se encontram espalhadas na região da cidade de Poços de Caldas, Minas Gerais, Brasil. Posicionando-as em contato direto com o equipamento, assim como os elementos anteriores, verifica-se o espectro da radiação gama em função da energia (Fig. 6).

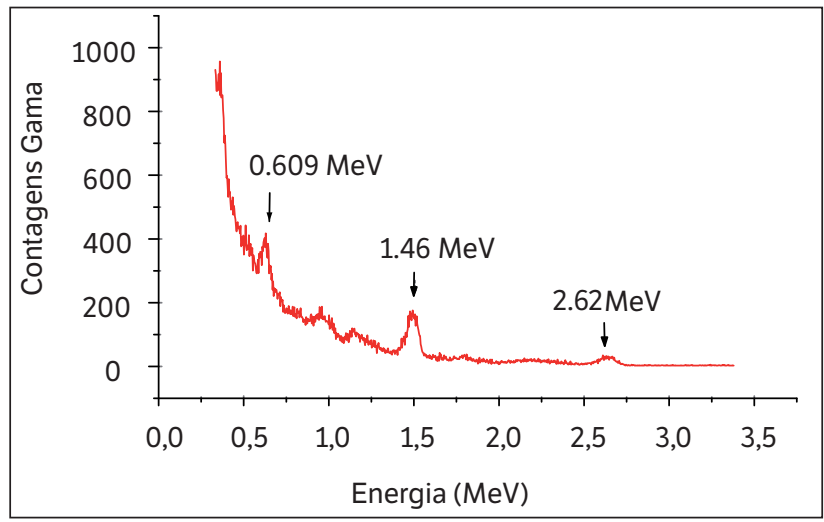

Figura 5: Gráfico da medição de radiação emitida pelas fontes radioativas Sr-90 e Po-210 e pelo meio ambiente.

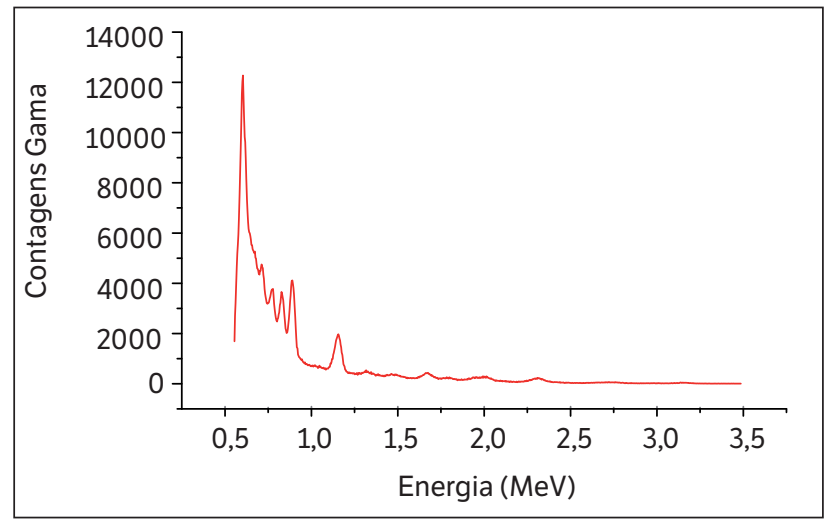

Figura 6: Espectro de amostras de uranitas da região de Poços de Caldas, Minas Gerais.

A análise dos gráficos das Figs. 1 e 2 evidenciou a radiação ambiental presente na região do campus do ITA, com destaque nos picos de 0,609 $\mathrm{MeV}\left({ }^{214} \mathrm{Bi}\right), 1,10 \mathrm{MeV}\left({ }^{214} \mathrm{Bi}\right), 1,46 \mathrm{MeV}$ $\left({ }^{40} \mathrm{~K}\right), 1,76 \mathrm{MeV}\left({ }^{214} \mathrm{Bi}\right)$ e 2,62 MeV $\left({ }^{232} \mathrm{Th}\right)$. No gráfico da Fig. 4, o elemento radioativo Cs-137 em contato com o detector emitiu uma intensidade elevada de raios gama, ofuscando a radiação ambiental presente no local.

O gráfico da Fig. 5 mostra que os elementos radioativos Sr-90 e Po-210, que emitem partículas alfa e beta, não ocasionaram mudança. A radiação alfa é barrada facilmente por uma folha de papel, a beta, por uma chapa de alumínio, e a gama por uma chapa grossa de chumbo, conforme ilustra a Fig. 7. Como o detector utilizado neste trabalho é protegido por uma camada fina de alumínio, não é possível executar medidas das radiações alfa e beta. 


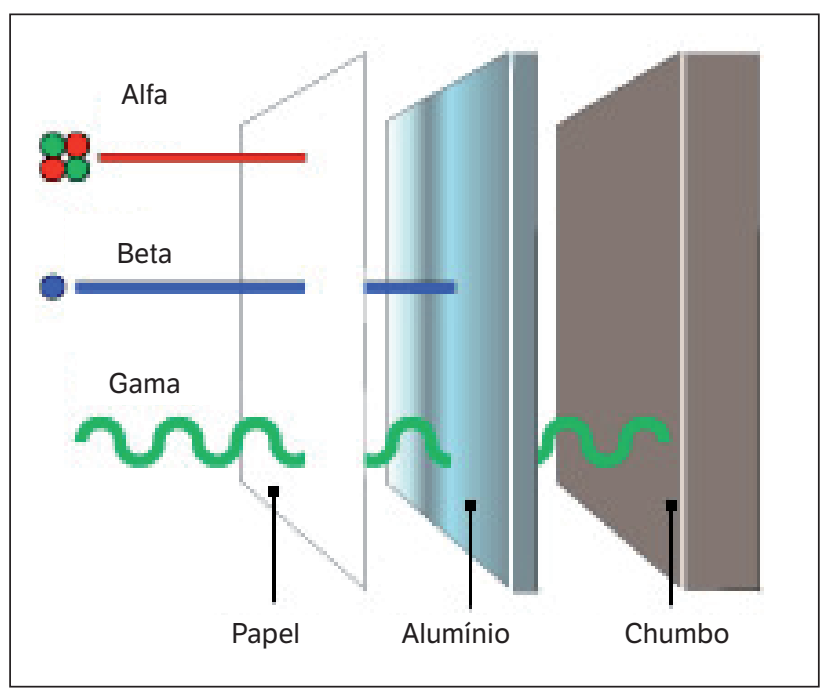

Figura 7: Esquema demonstrativo sobre transmissão e absorção das radiações empregadas neste trabalho?.

\section{CONCLUSÃO}

Foram realizadas, no campus do Instituto Tecnológico de Aeronáutica, São José dos Campos, São Paulo, Brasil, quatro medidas da radiação gama natural presente na interface soloatmosfera (considerando os espectros da intensidade $\times$ energia). $\mathrm{Na}$ primeira delas, foi obtido o espectro da radiação ambiente sem intervenção humana. Em seguida, foram empregadas fontes radioativas alfa (Po-210), gama (Cs-137, que fornece raios gama em $0,662 \mathrm{keV}$ e $1,17 \mathrm{MeV}$ ) e beta (Sr-90). Na terceira medida, foi retirado o Cs-137, obtendo-se somente o espectro da radiação ambiente, pois as partículas alfa e beta não penetram a camada de alumínio presente no detector. Por último, foi realizada a medida da radiação emitida pelas pedras com urânio, com picos de energia dominante entre 0,5 e 1,10 MeV. Com essas análises é possível verificar o perfeito funcionamento do detector, evidenciando os principais picos de foto-absorção existentes em cada medida. Pode-se ressaltar que o equipamento utilizado é totalmente portátil possibilitando detectar os espectros de radiação gama em diversas regiões do Brasil em qualquer período do ano.

\section{REFERÊNCIAS}

1. Martin IM, Gomes MP, Sismanoglu BN, Santos TA, Corrêa JP. Monitoring and ionizing radiation using Geiger instrument during February to April 2015 in Sao Jose dos Campos, SP, Brazil. J. Environ. Science and Engeneering. 2015;A(4):282-285. http:// doi.org/10.17265/2162-5298/2015.06.002

2. Hazardous isotopes [internet]. Buckten: Radioactivity and Radiation; 2007. [Acesso em 2016 Nov. 23]. Disponível em: http://www.geigercounter.org/radioactivity/isotopes.htm

3. Nelson G, Reilly D. Gamma-ray Interactions with matter. Passive nondestructive analysis of nuclear materials. 1991:27-42. Disponível em: http://www.lanl.gov/orgs/n/n1/panda/00326397

4. Aware coincidence box. Wilmington: Aware Electronic Corp. [Acesso em 2016 Nov. 25]. Disponível em: www.aw-el.com

5. Gusev AA, Martin IM, Alves MA, Abreu AJ. Simulation of the Radiation fallout from gamma-ray mesurements. Model. Earth Syst. Environ. 2015;1:18. https://doi.org/10.1007/s40808-0150016-3

6. Martin IM, Gomes MP, Ferro MAS, Pinto MLA, Antônio FC. Mesurements of X-ray and gamma radiation at ground level and their correlation with atmospheric electric discharges and rainfall in São José dos Campos, SP, Brazil.World Environment. 2013;3(4):138-141. http://doi.org/10.5923/j.env.20130304.03

7. Silva ALS. Radiação gama [internet]. Florianópolis: Info Escola; 2013. [Acesso em 2016 Nov. 25]. Disponível em: https://www. infoescola.com/fisica-nuclear/radiacao-gama 